\title{
On being human in the face of a pandemic
}

\author{
As the COVID-19 pandemic sweeps through the world, we must reassess the principles that guide our individual and \\ collective responses and the way we operate in society. In the face of crisis, we must lead with science and humanity.
}

t has been three short months since a pneumonia of unknown cause was first reported in the city of Wuhan in the Hubei province of China. Yet at the time of this writing, in early April 2020, the number of COVID-19 cases is nearing 1.5 million worldwide, with $>87,000$ fatalities and over 3 billion people under some form of stay-at-home orders. Pandemics are certainly not a new occurrence in human history, with the H1N1 influenza pandemic of 2009 being particularly fresh in memory. The quality and sophistication of modern medicine and technology are at their peak. Furthermore, we live in an era of rapid and efficient global communication and collaboration of nations. And yet the causative coronavirus, SARS-CoV-2, is sweeping through the world with a devastating death toll, bringing healthcare systems to a breaking point, grounding half the global population and shattering our perceptions of normality. The forensic analysis of how we got to this point will have to wait while we fight this crisis. Suffice it to say that the combination of a rapidly disseminating new virus and a toxic mix of political vacillation and opportunism, misinformation, weak health systems and science denialism created the perfect storm.

This pandemic has been an urgent wake-up call for many high-income countries, as it has brought to the fore the fragility of their healthcare systems, not because their otherwise well-equipped and well-staffed hospitals did not have stockpiles of thousands of ventilators, but because they failed to mount a rapid and decisive response to protect their citizens on a political level. The lack of clear, science-driven guidance, effective testing and early planning to procure the necessary medical equipment despite the mounting evidence that we were facing a global health emergency has been especially shocking in the USA, EU and UK. Despite priding themselves on the power of their respective unions, quality of science and medical expertise, and economic strength, they each failed spectacularly in their initial response to the pandemic, coming across at best as unprepared and at worst as naïve and uninformed. This lack of preparedness has unfortunately placed vulnerable parts of the population at greater risk, not just of contracting this infection but also of potentially being unable to receive essential care for other serious conditions, including cancer, should hospitals become inundated with patients with COVID-19.

With powerhouses such as the USA, EU and UK floundering and unable to coordinate a unified, effective response, how lower-income countries with already compromised health systems will face this challenge and whether the necessary international support will be forthcoming have become pressing questions.

Yet some continue to characterize this virus as 'the great equalizer'. The facts say it is nothing of the sort. Beyond the differences in how people respond to the infection itself, COVID-19 has accentuated the differences between the haves and the have-nots. As part of the population shelters in place in comfortable homes, many others reside in cramped living spaces or are homeless. While many of us have been readjusting to a work-from-home reality and may be worrying about stalled careers and missed professional opportunities, many others are essential workers who now face increased risks to keep key services up and runningnot to mention the large numbers of people who are losing their livelihoods as the economy continues to crash, or those who were already relying on external help to get by. Moreover, curtailing social activities and human interaction has revealed the gulf between those who, surrounded by their family, are anxious to keep them safe, and those who are alone and have been left even more isolated. While the needs and anxieties of every person are valid, it is essential to recognize that certain parts of the population bear a disproportionate burden of this crisis and need our support and protection as a priority. Unfortunately, the pervasive view, especially at earlier stages of the pandemic, that only those with underlying conditions or of older age were vulnerable, revealed instead an alarming degree of ableism and ageism in our society. Stories abound of people who, lacking a modicum of social responsibility or self-control, refused to follow social-distancing guidelines and thereby endangered themselves and others. More disturbing, however, have been the state-level ideas of relying on the development of naturally acquired herd immunity or of focusing on jumpstarting the economy before achieving outbreak containment. Both strategies would come at a huge cost of human life, given the current absence of reliable point-of-care testing to distinguish carriers from recovered or healthy people.

At the opposite end of the spectrum lies the response of those in the medical and scientific communities, who have been a beacon of solidarity, resilience and action. The doctors, nurses and all other healthcare workers who, faced with unprecedented challenges, are bearing the brunt of this pandemic on the front lines have been nothing short of inspiring. Moreover, despite the fact that the routine research enterprise has come to an abrupt halt, with many laboratories in several countries now closed, researchers have also rallied to fight the pandemic. Scientists from around the world have rapidly diverted efforts toward all aspects of COVID-19 work, from basic biology and immunology to diagnostic testing, vaccines and therapeutic interventions. Volunteers have been offering their skills as part of international collaborations, and shuttered labs have been donating reagents and essential lab and personal protective equipment. Others have been making the most of their digitally confined existence by applying their expertise to parse the rapidly expanding SARS-CoV-2 literature, distilling and disseminating knowledge to other scientists and the general public. If there is a silver lining in this challenging situation, it is seeing this community spirit flourish and also experiencing the shift of an often science-skeptic public, who are now turning to scientists and doctors with renewed trust. It is essential to maintain this renewed focus by the public and governments on science, medicine and technology even after the threat of COVID-19 is addressed.

"Man is the measure of all things," said the ancient Greek philosopher Protagoras. Setting aside the philosophical debate that surrounds this anthropocentric view of the universe, the message is particularly apt today, when the world is in the throes of the COVID-19 pandemic. Each human experience, and by extension each human life, is unique, equally valid and precious. During these tumultuous times, we need to remember this and join efforts to lead with science and humanity.

Published online: 9 April 2020 https://doi.org/10.1038/s43018-020-0062-2 